\title{
Thermal Error Modelling for Real-Time Error Compensation
}

\author{
J. S. Chen, J. Yuan and J. Ni \\ Department of Mechanical Engineering and Applied Mechanics, The University of Michigan, Ann Arbor, USA
}

\begin{abstract}
A modelling strategy for the prediction of both the scalar and the position-dependent thermal error components is presented. Two types of empirical modelling method based on the multiple regression analysis (MRA) and the artificial neural network $(A N N)$ have been proposed for the real-time prediction of thermal errors with multiple temperature measurements. Both approaches have a systematic and computerised algorithm to search automatically for the nonlinear and interaction terms between different temperature variables. The experimental results on a machining centre show that both the MRA and the ANN can accurately predict the time-variant thermal error components under different spindle speeds and temperature fields. The accuracy of a horizontal machining centre can be improved through experiment by a factor of ten and the errors of a cut aluminium workpiece owing to thermal distortion have been reduced from $92.4 \mu \mathrm{m}$ to $7.2 \mu \mathrm{m}$ in the lateral direction. The depth difference due to the spindle thermal growth has been reduced from $196 \mu \mathrm{m}$ to $8 \mu \mathrm{m}$.
\end{abstract}

Keywords: Accuracy; CNC machine tools; Error compensation; Thermal error modelling

\section{Introduction}

Research on thermal error compensation for machine tools by both industries and academic institutions has been accelerated recently in response to the increasing demand for high-quality products. Effective compensation relies on the accurate prediction of the time-variant thermal errors during machining. Since the thermal errors of machine tools exhibit highly nonlinear interactions [1], a precise quantitative prediction is difficult to achieve by a theoretical analysis.

The quantitative prediction of thermal errors is usually achieved by empirical modelling approaches which correlate machine thermal errors to temperature measurements of the machine through experiments and data analysis techniques.

Correspondence and offprint requests to: Professor J. Ni, Department of Mechanical Engineering and Applied Mechanics, University of Michigan, 3424 G.G. Brown Building, Ann Arbor, MI 48109-2125, USA.
Most of the previous efforts of predicting thermal errors by an empirical modelling approach have focused on solving one specific problem such as the spindle growth [2-4] or leadscrew thermal expansion [5]. Recently, progress has been made in compensating for the thermal planar and volumetric errors [6,7]. In Chen et al. [6], the time-variant volumetric error (including both geometric and thermal errors) of a 3D machine tool was synthesised from 32 error components existing at machine elements. The accuracy of the compensation thus relies on the modelling accuracy of the error components.

One problem for a $2 \mathrm{D}$ or $3 \mathrm{D}$ thermal error compensation is that some thermal errors such as the positioning and angular errors of a movable slide are not only temperature-dependent but also slide-position dependent. The empirical model developed should be capable of accommodating both the temperature and position changes during machining.

Another problem is that most previous studies use only one temperature variable of the machine to predict a thermal error. However, thermal errors are sensitive to varying spindle speeds and the temperature history (warm-up or cool-down cycles) owing to different thermal time constants of various machine components, as shown in Chen et al. [6]. For example, the thermal growth of a spindle is contributed to not only by the thermal expansion of the spindle itself but also by the thermal bending of the machine column. The spindle heats up and cools down quickly but the column has a relatively sluggish response to generated heat. Therefore, an empirical model using multiple temperature variables is necessary in order to include the effects of different thermal time constants and the interaction between different temperature variables. Although a multiple regression model for thermal error prediction has been reported in Donmez et al. [7] and Kurtoglu [5], there is no systematic algorithm using statistical criteria for the selection of positions, orders and cross-product terms of the temperature measurements.

The objective of this paper is to report a new modelling strategy developed for both the scalar and the slide-positiondependent error components. Two multivariable empirical models based on multiple regression analysis (MRA) and an artificial neural network (ANN) have been used to fit the thermal error components. Both the MRA and the ANN use a systematic algorithm to search for the nonlinear and interaction characteristics of thermal errors based on statistical 
criteria. The MRA approach uses a stepwise regression analysis to select the positions, orders and cross-product terms of temperature measurements. In the ANN method, the nonlinear and interaction characteristics of thermal errors are recognised by a backpropagation training procedure. Some experiments have been carried out for both model-fitting and model-checking purposes. The prediction accuracy of new observations, noise resistance robustness, and fault tolerance of the MRA and the ANN methods have also been compared.

\section{Basic Forms of the Error Component Models}

As presented in Chen et al. [6], the thermal volumetric error, which is the relative error between the movements of the tool and the workpiece, was decomposed into 32 error components existing at machine elements. A kinematic model for a $3 \mathrm{D}$ machine such as a horizontal machining centre was also developed to synthesise these 32 error components in order to predict the volumetric error at any location within the machine working zone. In addition to the well-known 21 errors which include 3 translational and 3 rotational errors of each slide and 3 squareness errors between machine axes, 11 additional thermal error components were identified. The first group of the additional thermal errors accounts for thermal drifts at the tool-tip which include three translational drifts of the spindle and two inclinational drifts of the spindle axis. The second group of the additional thermal errors is due to thermal shifts at the references of machine axes which include 6 translational errors if one axis is selected as the reference for two other axes.

The 32 thermal error components can be classified into two types: the scalar errors and the position-dependent errors. The squareness errors and the 11 thermal drifts at the spindle and at the references of machine axes are regarded as the scalar errors, because they can be represented as a timevariant but scalar formulation. For every movable slide, the three translational and three rotational errors could also vary with the travel of the slide. Therefore, these errors are not only temperature dependent but also position dependent.

\subsection{Scalar Thermal Error Component}

The basic strategy for modelling the thermal error components is to separate the errors into two parts:

1. Geometric error part, which exists under an evenly distributed reference temperature $\left(20^{\circ} \mathrm{C}\right.$ or other prescribed temperature).

2. Thermal error part, which is an additional error to the geometric error owing to the change of the machine temperature field.

For the scalar thermal errors the basic form of the model is:

$$
\delta_{\mathrm{s}}(t)=\delta_{\mathrm{g}}+f_{\mathrm{t}}\left(\Delta T_{1}, \Delta T_{2}, \ldots, \Delta T_{n}\right)
$$

where $\delta_{s}(t)=$ the time-variant scalar error $\delta_{\mathrm{g}}=$ the geometric part of the scalar error
$f_{1}\left(\Delta T_{1}, \Delta T_{2}, \ldots, \Delta T_{n}\right)=$ the thermal part of the scalar error as a function of the machine temperature field

$\Delta T_{1}, \Delta T_{2}, \ldots, \Delta T_{n}=$ temperature changes of machine structures at points $1,2, \ldots, n$

\subsection{Position-Dependent Thermal Error Component}

Observations from a variety of experiments have shown that although the slide position-dependent errors varied with temperatures, their basic profiles along the travels did not change drastically and only the slopes differed, as shown in Fig. 1. This phenomenon applies to both translational and angular errors. The 18 slide position-dependent errors are then formulated as the summation of the stationary error profiles and time-variant slopes. The stationary error profiles (i.e. geometrically induced errors) exist under machine coldstart conditions. During operations, the time-variant slopes (i.e. thermally-induced errors) are added to the geometricallyinduced errors.

Therefore, for the position-dependent errors, the mathematical formulation becomes

$$
\begin{aligned}
\delta_{\mathrm{p}}(p, t) & =f_{\mathrm{g}}(x, y, z)+k_{1}(t) p+k_{2}(t) p^{2}+\ldots \\
\text { and } k_{i}(t) & =f_{t_{i}}\left(\Delta T_{1}, \Delta T_{2}, \ldots, \Delta T_{n}\right) \quad(i=1,2, \ldots, m)(3)
\end{aligned}
$$

where $\delta_{\mathrm{p}}(p, t)=$ the time-variant and position-dependent error

$f_{\mathrm{g}}(x, y, z)=$ the geometric error part of the positiondependent error

$p=$ position or travel of a slide

$k_{1}(t), k_{2}(t)=$ the thermal-variant coefficients of the positiondependent error

$f_{t_{i}}\left(\Delta T_{1}, \Delta T_{2}, \ldots, \Delta T_{n}\right)=$ the coefficients as functions of the temperature field

$m=$ the order of the thermal-variant position-dependent error

Notice that the geometric part of the slide-position dependent errors, $f_{\mathrm{g}}(x, y, z)$, is not only a function of the coordinate of the slide being measured but also a function of the other two coordinates. This can, thus, accommodate the non-rigid body kinematic effect, Chen et al. [8].

For the thermal error part, this new approach monitors the slope variations of error profiles and reduces the number of

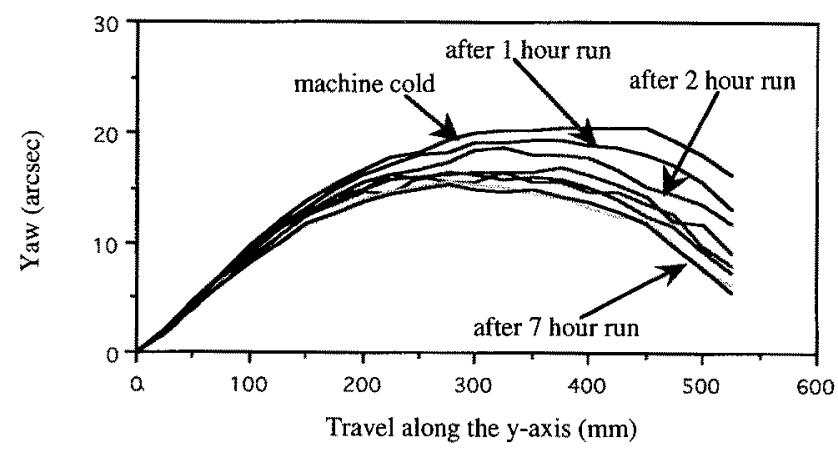

Fig. 1. The thermal variation of the yaw angular errors of the $y$-axis. 
empirical models needed for predicting position-dependent errors. In most cases, the position-dependent errors can be approximated accurately with only the first-order term.

Two different methods will be presented in the following sections to construct the empirical model $f_{t_{i}}\left(\Delta T_{1}, \Delta T_{2}, \ldots\right.$, $\Delta T_{n}$ ) by multiple regression analysis and by using an artificial neural network.

\section{Multiple Regression Analysis (MRA) Approach}

\subsection{Principle of the MRA for Thermal Error Component Modelling}

The proposed multiple regression model for $f_{t_{i}}\left(\Delta T_{1}, \Delta T_{2}\right.$, $\left.\ldots, \Delta T_{n}\right)$ is:

$$
\begin{aligned}
& f_{t_{i}}\left(\Delta T_{1}, \Delta T_{2}, \ldots, \Delta T_{n}\right)=\left\{A_{1}\right\}^{\prime}\{\Delta T\} \\
& +\{\Delta T\}^{\prime}\left[A_{2}\right]\{\Delta T\}
\end{aligned}
$$

where $\left\{A_{1}\right\},\left[A_{2}\right]=$ coefficient vector and matrix of the regression model respectively

$\{\Delta T\}=$ temperature rise vector of machine structures

One of the most difficult problems in regression analysis is the selection of the set of independent variables to be used in the model. It is important that any key variables that could affect the ability of the model to predict a new observation should not be neglected. However, a regression analysis with a large number of independent variables is costly. It may also cause a serious round-off problem in estimating the model coefficients using a least-squares method if some highly intercorrelated independent variables exist.

Independent variables were initially screened out based on experience and the results of a statistical correlation analysis. However, typically, the number of independent variables that remained after this initial screening was still large. The stepwise regression analysis with a computerised automatic search algorithm was used for a second screening. This search method develops a sequence of regression models; at each step adding or deleting a new variable using an F-test or other statistical criteria.

\subsection{Modelling Scalar Errors Using the MRA}

To demonstrate the modelling of the scalar errors using the MRA approach, the spindle thermal drifts were considered. During experiments, more than 20 thermocouples were used to monitor the machine temperatures. Nine temperature measurements which had strong correlations with thermal drifts of the spindle were selected after the first screening (see Table 1). The nine temperature measurements were further classified into three subgroups corresponding to the three directions of spindle thermal drifts (Table 2).

A second-order regression model, which included the firstand second-order terms of temperature rise and also the interaction terms between any two temperature measurements, was used to fit the spindle thermal drifts. The numbers of
Table 1. Selected temperature measurement and thermocouple positions.

\begin{tabular}{ll}
\hline Symbol & Purpose and position of thermocouples \\
\hline$\Delta T_{1}$ & Variation of the environmental temperature \\
$\Delta T_{2}$ & Variation of the spindle housing temperature \\
$\Delta T_{3}$ & Variation of the y-axis nut temperature \\
$\Delta T_{4}$ & Variation of the $y$-axis bearing temperature \\
$\Delta T_{5}$ & Variation of the average column temperature \\
$\Delta T_{6}$ & Variation of the front-right side of column \\
$\Delta T_{7}$ & Variation of the front-left side of column \\
$\Delta T_{8}$ & Variation of the rear-right side of column \\
$\Delta T_{9}$ & Variation of the rear-left side of column \\
\hline
\end{tabular}

Table 2. Subgroups for spindle thermal drift components.

Spindle thermal Subgroups of monitored temperature drifts

\begin{tabular}{ll}
$\Delta x$ & $\Delta T_{1}, \Delta T_{2}, \Delta T_{3}, \Delta T_{6}-\Delta T_{7}, \Delta T_{8}-\Delta T_{9}$ \\
$\Delta y$ & $\Delta T_{1}, \Delta T_{2}, \Delta T_{3}, \Delta T_{4}, \Delta T_{5}, \Delta T_{6}-\Delta T_{8}, \Delta T_{7}-\Delta T_{9}$ \\
$\Delta z$ & $\Delta T_{1}, \Delta T_{2}, \Delta T_{3}, \Delta T_{6}-\Delta T_{8}, \Delta T_{7}-\Delta T_{9}$ \\
\hline
\end{tabular}

unscreened coefficients were 20,35 and 20 in the $x$-, $y$ - and $z$-directions, respectively. The stepwise regression analysis was applied to further reduce the number of coefficients.

For the stepwise regression analysis, 810 data sets containing spindle thermal drifts and temperature fields mentioned in Table 1 were collected. The 810 observation sets were measured from 5 different tests with spindle speeds at 600 r.p.m., 1000 r.p.m., 1500 r.p.m., 2100 r.p.m. and 2600 r.p.m. In each of the above tests, the machining centre was run for 6 hours to warm-up and then stopped for 10 hours to allow the machine to cool down.

Figure 2 is an example of the output results of the spindle thermal drift model in the $x$-direction using a commercial stepwise regression analysis package, SYSTAT 5.0. After the regression analysis, the number of coefficients was reduced to 7 . The fitted model for the thermal error in the $x$-direction is:

$$
\begin{aligned}
\Delta x= & -0.586^{*} \Delta T_{2}+7.527^{*} \Delta T_{3}-36.966^{*} \\
& \left(\Delta T_{6}-\Delta T_{7}\right)+10.809^{*}\left(\Delta T_{8}-\Delta T_{9}\right) \\
& -0.149^{*}\left(\Delta T_{3}\right)^{2}-18.119^{*}\left(\Delta T_{6}-\Delta T_{7}\right)^{2} \\
& +2.499^{*} \Delta T_{2}^{*}\left(\Delta T_{6}-\Delta T_{7}\right)
\end{aligned}
$$

The spindle thermal drift models in the $y$ - and z-directions were determined similarly and the numbers of coefficients were reduced to 15 and 7, respectively. Figures 3-5 compare the experimental results and the predicted results. They show that the three regression models can fit the thermal drifts of the spindle closely.

\subsection{Modelling Position-Dependent Errors Using the MRA}

To demonstrate the method of modelling position-dependent thermal errors by MRA, the positioning errors of the $y$-axis 


\begin{tabular}{|c|c|c|c|c|}
\hline \multicolumn{5}{|c|}{ STEPWISE REGRESSION WITH ALPHA-TO-ENTER $=.001$ AND ALPHA-TO-REMOVE $=015$} \\
\hline STEP $=1$ & ENTER & $\Delta \mathrm{T} 2$ & $\mathrm{R}=.751$ & RSQUARE $=.564$ \\
\hline STEP $=2$ & ENTER & $\Delta \mathrm{T} 5^{*} \Delta \mathrm{T} 5$ & & RSQUARE $=.609$ \\
\hline STEP $=3$ & ENTER & $(\Delta T G-\Delta T 8)$ & & RSQUARE $=.726$ \\
\hline$S T E P=4$ & ENTER & $\Delta T_{3}$ & & RSQUARE $=862$ \\
\hline$S T E P=5$ & ENTER & $\Delta \mathrm{rs}$ & & RSQUARE $=.897$ \\
\hline STEP $=6$ & ENTER & $(\Delta \mathrm{T} 7-\Delta \mathrm{T} 9)$ & & RSQUARE $=908$ \\
\hline STEP $=7$ & ENTER & $\Delta \mathrm{T}^{*} \Delta \mathrm{T} 5$ & & RSQUARE $=.913$ \\
\hline STEP $=8$ & ENTER & $\Delta \mathrm{T} 3 * \Delta \mathrm{T} 3$ & & RSQUARE $=.946$ \\
\hline$S T E P=9$ & REMOV! & & & RSQUARE $=.945$ \\
\hline STEP $=10$ & ENTER & $\Delta \mathrm{T}_{2}^{*} \Delta \mathrm{T} 2$ & & RSQUARE $=948$ \\
\hline$S T E P=11$ & ENTER & $\Delta T^{*} \Delta T 3$ & & RSQUARE $=.950$ \\
\hline STEP $=12$ & ENTER & $\Delta \mathrm{T}^{*}(\Delta \mathrm{T} 7-\Delta \mathrm{T} 9)$ & & RSQUARE $=.953$ \\
\hline STEP $=13$ & REMOV & $\mathrm{E} \Delta \mathrm{T} \mathrm{T}^{*} \Delta \mathrm{T} \mathrm{T} 3$ & & RSQUARE $=.952$ \\
\hline STEP= 14 & ENTER & $\Delta \mathrm{T}^{*}{ }^{*}(\Delta \mathrm{T} 6-\Delta \mathrm{T} 8)$ & & RSQUARE $=.953$ \\
\hline STEP= 15 & ENTER & $\Delta T 4^{*}(\Delta T 7-\Delta T 9)$ & $R=.978$ & RSQUARE $=.956$ \\
\hline STEP $=16$ & REMOV & $E \Delta T 3 *(\Delta T 7-\Delta T 9)$ & $R=.978$ & RSQUARE $=.956$ \\
\hline STEP $=17$ & ENTER & $\Delta \mathrm{T} 4 * \Delta \mathrm{T} 4$ & $\mathrm{R}=.980$ & RSQUARE $=.961$ \\
\hline STEP $=18$ & ENTER & $\Delta \mathrm{TS}$ & $R=.981$ & RSQUARE $=.963$ \\
\hline STEP = 19 & ENTER & $\Delta \mathrm{T} 4$ & $R=.983$ & RSQUARE $=965$ \\
\hline STEP $=20$ & ENTER & $\Delta \mathrm{T} 4 * \Delta \mathrm{TS}$ & $\mathrm{R}=.983$ & RSQUARE $=.966$ \\
\hline$S T E P=21$ & ENTER & $(\Delta \mathrm{T} 6-\Delta \mathrm{T} 8)^{*}(\Delta \mathrm{T} 6-\Delta \mathrm{T} 8)$ & $R=.983$ & RSQUARE $=.967$ \\
\hline $\mid$ STEP $=22$ & $\begin{array}{l}\text { ENTER } \\
\text { FETTR }\end{array}$ & $(\Delta \mathrm{TT} 6-\Delta \mathrm{TT} B)^{*}(\Delta \mathrm{TT}-\mathrm{\Delta TT})$ & $R=.985$ & $\begin{array}{l}\text { RSQUARE }=.970 \\
\text { PSOUARF }=973\end{array}$ \\
\hline $\begin{array}{l}S T E P=23 \\
S T E P=24\end{array}$ & ENTER & $(\Delta \mathrm{TT}-\Delta \mathrm{TT} 9)^{*}(\Delta \mathrm{TT}-\Delta \mathrm{T} 9)$ & $\begin{array}{l}\mathrm{R}=986 \\
\mathrm{R}=986\end{array}$ & $\begin{array}{l}\text { RSQUARE }=.973 \\
\text { RSOUARE }=973\end{array}$ \\
\hline $\begin{array}{l}\text { STEP }=24 \\
\text { STEP }=25\end{array}$ & $\begin{array}{l}\text { REMOV } \\
\text { REMP }\end{array}$ & $\mathrm{E} \Delta \mathrm{AT} 5^{*} * \mathrm{TS}$ & $\begin{array}{l}\mathrm{R}=.986 \\
\mathrm{R}=986\end{array}$ & $\begin{array}{l}\text { RSQUARE }=9737 \\
\text { RSOUARE }=977\end{array}$ \\
\hline $\mid \begin{array}{l}S T E P=25 \\
S T E P=26\end{array}$ & $\begin{array}{l}\text { ENTER } \\
\text { REMOV }\end{array}$ & 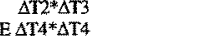 & $\begin{array}{l}R=.986 \\
R=986\end{array}$ & $\begin{array}{l}\text { RSQUARE }=.973 \\
\text { RSOUARE }=973\end{array}$ \\
\hline $\begin{array}{l}S T E P=26 \\
S T E P=27\end{array}$ & $\begin{array}{l}\text { REMOV } \\
\text { REMOV }\end{array}$ & 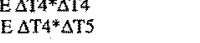 & $\begin{array}{l}R=.986 \\
R=.986\end{array}$ & RSQUARE $=.973$ \\
\hline \multicolumn{5}{|c|}{ THE SUBSET MODEL INCLUDES THE FOLLOWTNG PREDICTORS: } \\
\hline \multicolumn{5}{|c|}{$\begin{array}{l}\Delta \mathrm{T} 2, \Delta \mathrm{T} 3, \Delta \mathrm{T} 4,(\Delta \mathrm{TT} 6-\Delta \mathrm{T} 8),(\Delta \mathrm{TT}-\Delta \mathrm{TT} 9), \Delta \mathrm{TS}, \Delta \mathrm{T} 2^{*} \Delta \mathrm{T} 2,(\Delta \mathrm{T} 6-\Delta \mathrm{T} 8)^{*}(\Delta \mathrm{T} 6-\Delta \mathrm{T} 8), \\
(\Delta \mathrm{TT}-\Delta \mathrm{TT})^{*}(\Delta \mathrm{TT}-\Delta \mathrm{T} 9), \Delta \mathrm{TT} 2^{*}(\Delta \mathrm{T} 6-\Delta \mathrm{T})\end{array}$} \\
\hline \multicolumn{5}{|c|}{ USE THESE PREDICTORS IN A NEW MODEL SENTENCE TO ESTTMATE THE COEFFICIENTS } \\
\hline \multicolumn{5}{|c|}{ 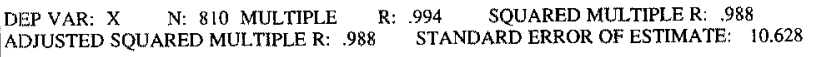 } \\
\hline VARLABLE & & OEFFCIENT & VARIABLE & COEFHCIENT \\
\hline & & -0.586 & $\Delta \mathrm{T}^{*} \Delta \mathrm{T} 3$ & -0.149 \\
\hline$\Delta \mathrm{T3}$ & & 7.527 & $(\Delta \mathrm{T} G-\Delta \mathrm{T} T)^{*}(\Delta \mathrm{T} 6$ & \\
\hline$\Delta \mathrm{Tt}-\Delta \mathrm{T} 7$ & & $\begin{array}{l}-36.966 \\
10.809\end{array}$ & $\Delta \mathrm{T}^{*}(\Delta \mathrm{T} 6-\Delta \mathrm{T})$ & \\
\hline
\end{tabular}

Fig. 2. The output of the regression analysis from SYSTAT 5.0 (spindle thermal drift in the $x$-direction).

were measured and analysed. Figure 6 shows the measured positioning errors of the $y$-axis under different machine thermal conditions. It is shown that the $y$-axis positioning errors depend not only on the machine temperatures but also on the $y$-axis position.
The time-invariant geometric component should be separated from the time-variant thermal component in order to use equation (2) to model these position-dependent thermal errors. As described in Section 2.2, the thermal component influences mainly the slope variation of the position-dependent thermal errors. The thermal status under the cold machine condition was taken as the reference status. The positioning errors in this thermal status were treated as "pure" geometric components. When the machine was warming up, the increase in the measured positioning errors, as compared with the geometric components, was treated as the contributions due to thermal error components. The geometric components, $f_{\mathrm{g}}$ $(x, y, z)$ in equation (2), was modelled by a polynomial function as follows:

$$
\begin{aligned}
f_{\mathrm{g}}(x, y, z)= & 2.424 \times 10^{-4}+3.096 \times 10^{-4} \\
& y-3.571 \times 10^{-6} y^{2}+1.474 \times 10^{-8} y^{3} \\
& -2.662 \times 10^{-11} y^{4}+1.738 \times 10^{-14} y^{5}
\end{aligned}
$$

To model the thermal error components, 51 sets of the slope variations of the $y$-positioning errors associated with the 5 temperature measurements $\left(\Delta T_{1}, \Delta T_{3}, \Delta T_{4}, \Delta T_{6}-\Delta T_{8}\right.$, $\Delta T_{7}-\Delta T_{9}$ ) shown in Table 2 , were collected. A second-order regression model with 20 unscreened coefficients was selected as the initial empirical model for the slope variations of the thermal positioning errors of the $y$-axis. After the stepwise regression analysis, the screened model contained only 4 coefficients as follows:

$$
\begin{aligned}
& k_{1}(t)=0.465^{*} \Delta T_{3}+2.705^{*} \Delta T_{4} \\
& \quad+3.675^{*}\left(\Delta T_{6}-\Delta T_{8}\right)-5.589^{*}\left(\Delta T_{7}-\Delta T_{9}\right)
\end{aligned}
$$

where $k_{1}(t)$ is the slope of the positioning error curve, as in equation (2). Note that the stepwise regression analysis suggested that there was no need to use higher-order and cross-product terms for modelling the $y$-axis positioning errors.

Substituting equations (6) and (7) into equation (2), the position-dependent thermal errors of the $y$-axis positioning accuracy were represented by the following model:

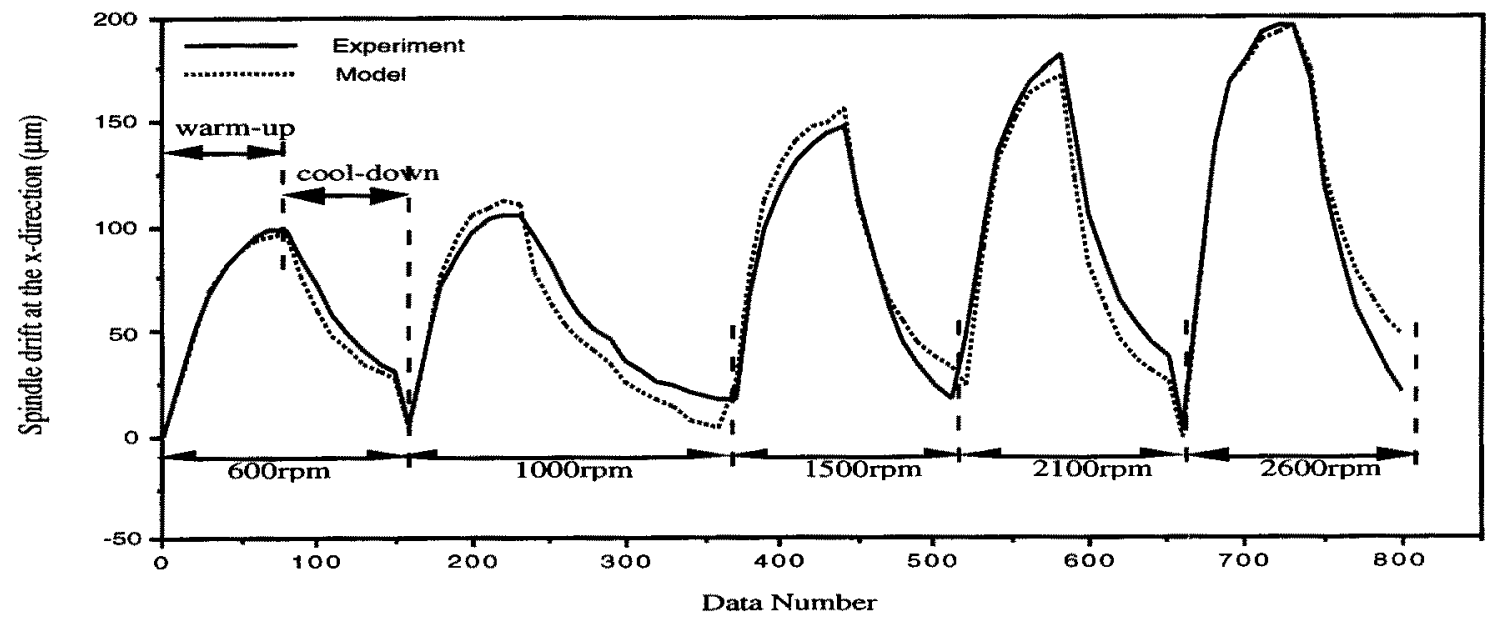

Fig. 3. The MRA curve-fitted result of the spindle drift in the $x$-direction. 


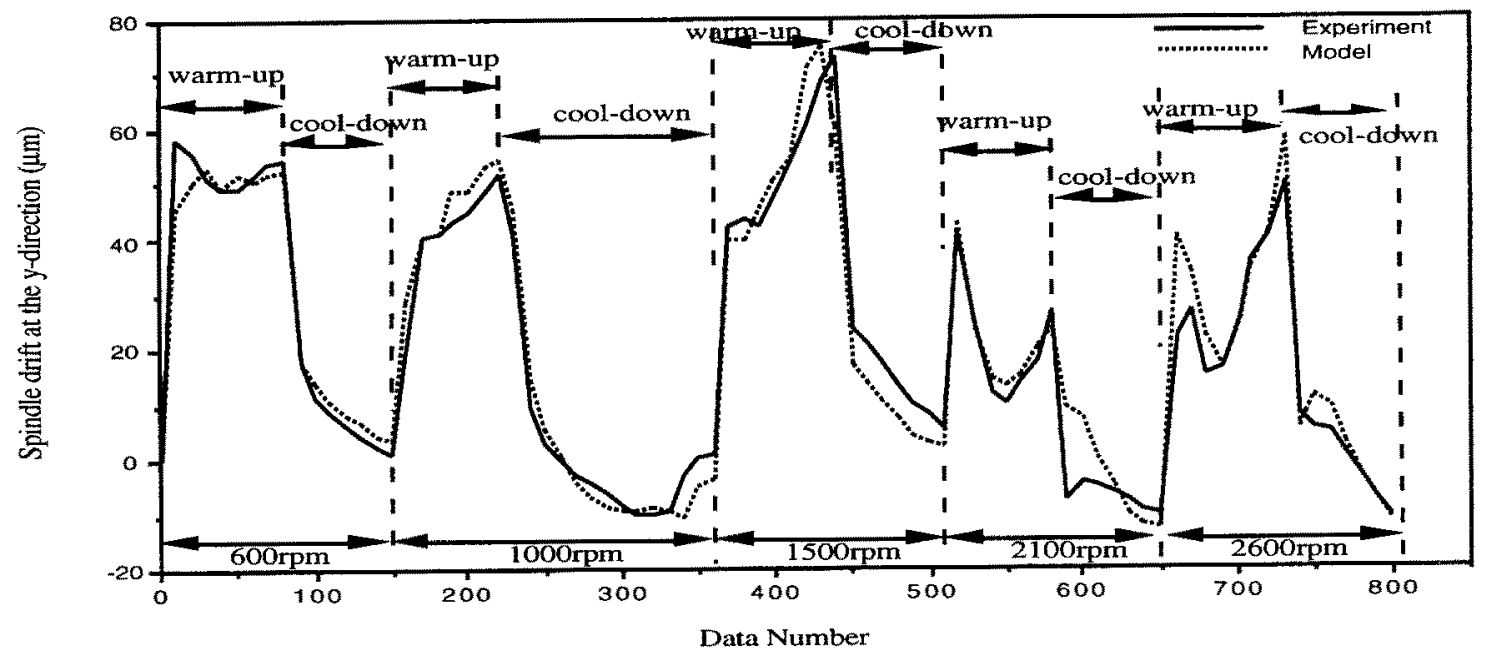

Fig. 4. The MRA curve-fitted result of the spindle drift in the $y$-direction.

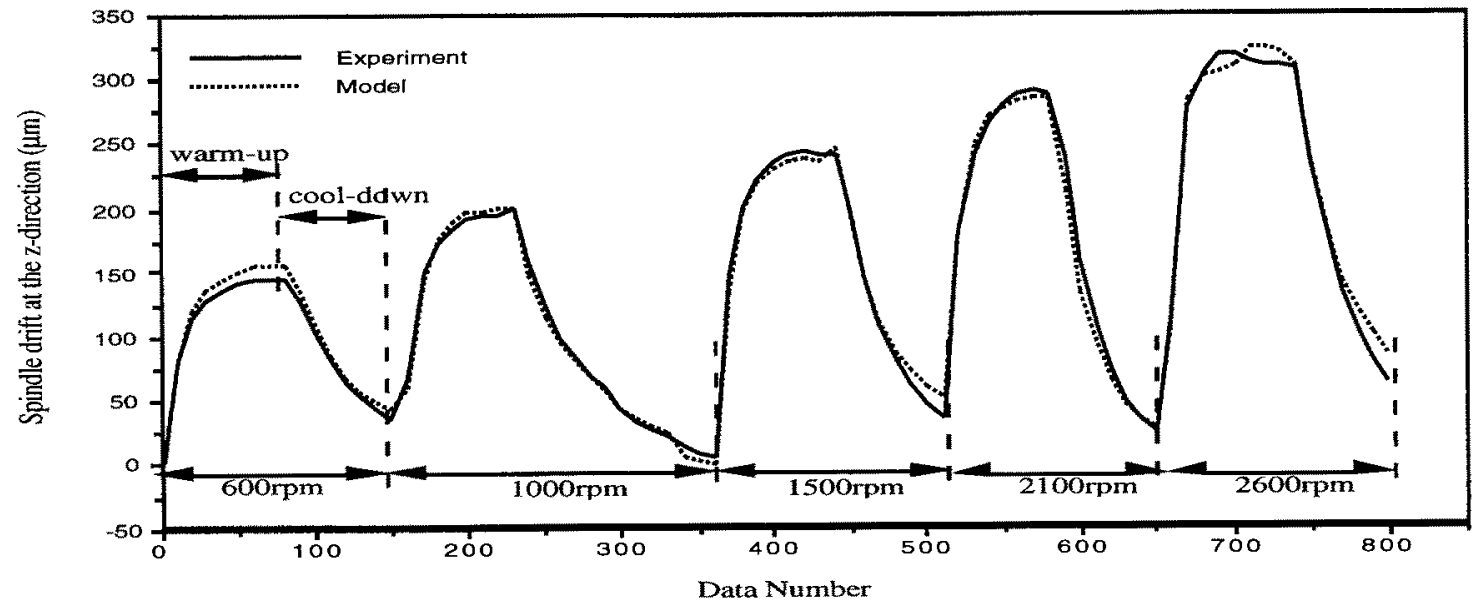

Fig. 5. The MRA curve-fitted result of the spindle drift in the $z$-direction.

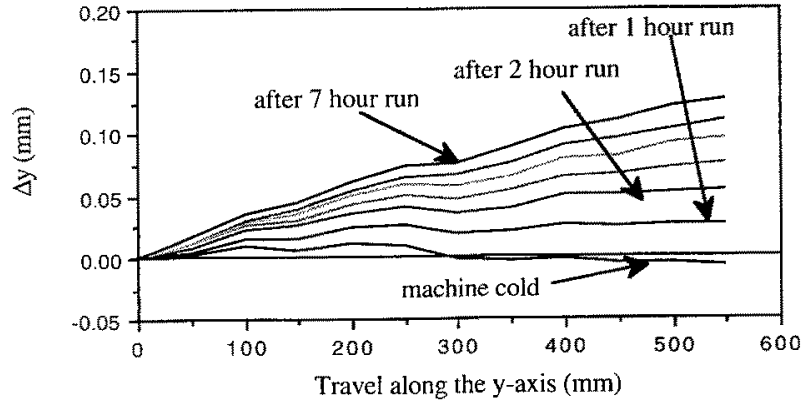

Fig. 6. The thermal variations of the $y$-positioning error.

$$
\begin{aligned}
& \delta_{y}(y, t)=2.424 \mathrm{e}^{-4}+3.096 \mathrm{e}^{-4} y-3.571 \mathrm{e}^{-6} \\
& y^{2}+1.474 \mathrm{e}^{-8} y^{3}-2.662 \mathrm{e}^{-11} y^{4} \\
& +1.738 \mathrm{e}^{-14} y^{5}+\left\{0.465 \Delta T_{3}\right. \\
& +2.705 \Delta T_{4}+3.675\left(\Delta T_{6}-\Delta T_{8}\right) \\
& \left.-5.589\left(\Delta T_{7}-\Delta T_{9}\right)\right\} \cdot y
\end{aligned}
$$

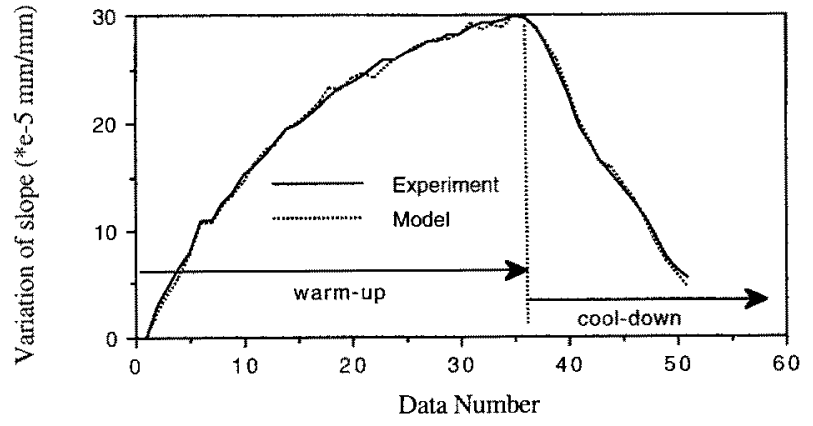

Fig. 7. The MRA curve-fitted result of the slope variation of the $y$ positioning error.

Figure 7 compares the experimentally measured slopes of the $y$-axis positioning errors and predicted slopes. It shows that the multiple regression analysis can closely fit the positiondependent errors during both the machine warm-up and cooldown cycles. 


\section{Artificial Neural Network (ANN) Approach}

\subsection{Principle of the ANN for Thermal Error Component Modelling}

An artificial neural network consists of two basic elements: the artificial neurons which process input information and the network which connects the artificial neurons. The artificial neuron receives a set of inputs and presents an output to other neurons. As shown in Figure 8, each input is multiplied by a corresponding weight. All of the weighted inputs are then summed and added to a threshold to determine the activation level of the neuron. That is,

$$
\mathrm{NET}=x_{1} w_{1}+x_{2} w_{2}+\ldots+x_{n} w_{n}+t h
$$

where NET $=$ summation of weighted inputs and threshold $x_{1}, x_{2}, \ldots, x_{n}=$ input signals received by an artificial neuron $w_{1}, w_{2}, \ldots, w_{n}=$ weights

th $=$ threshold

The weighted and combined inputs are further processed by an activation function $F$ to produce a nonlinear transformation of the inputs into the neuron's output. A "sigmoid" (meaning S-shaped) function is chosen as the activation function. This function is expressed mathematically as $F(x)$ $=1 /\left(1+\mathrm{e}^{-x}\right)$. Thus

$$
\mathrm{OUT}=F(\mathrm{NET})=\frac{1}{1+\mathrm{e}^{-\mathrm{NET}}}
$$

The sigmoid function has the important characteristic that it can adjust its gain automatically. For small signals the sigmoid function produces a high gain. As the magnitude of the signals become greater, the gain decreases. In this way, small signals are allowed to pass through without excessive attenuation if they are able to produce a usable output, while large signals can be accommodated by the network without saturation.

A multiple-layer feedforward network (usually called the multi-layer perceptron, MLP) is used to connect the artificial neurons. This kind of ANN has the architecture shown in Fig. 9. Its input layer is the input buffer for the temperature measurements of the machine and the output layer is the output buffer for the machine thermal errors. Layers between the input and the output buffers are called hidden layers. The functions of the hidden layers are to perform feature extraction and noise suppression between the input temperature measurements and output thermal errors. Although there can be more

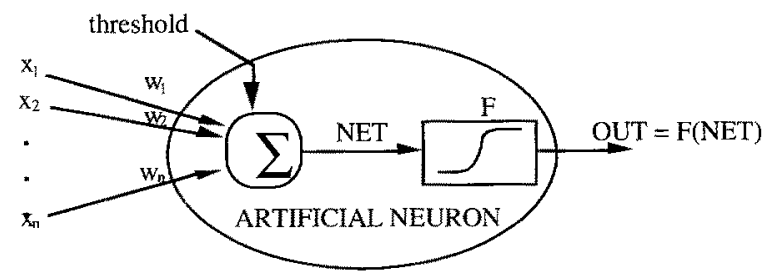

Fig. 8. The architecture of an artificial neuron.

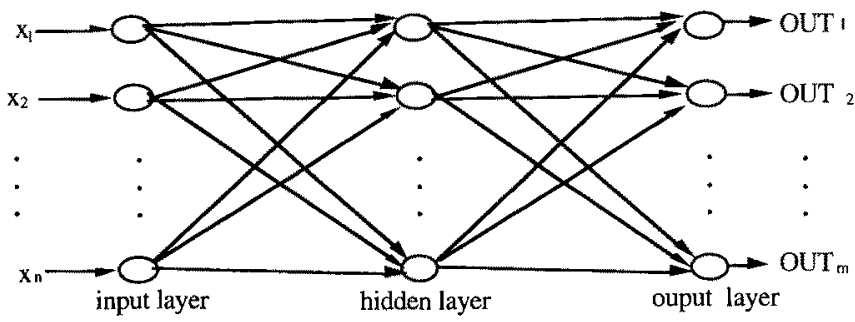

Fig. 9. A three-layer feedforward ANN for thermal error modelling.

than one hidden layer of an MLP, it has been shown that any nonlinear function between the inputs and the outputs can be mapped with one single hidden layer if the number of nodes in the hidden layer is sufficiently large.

For a multiple-layer perceptron ANN, the nonlinear and interaction characteristics of the thermal errors were represented in the form of connecting weights between neurons which were obtained through a supervised backpropagation training algorithm [9]. Supervised training requires the training pairing of each input vector with a target vector representing the desired output. Usually a network is trained by a number of such training pairs. The training pairs should include all of the patterns of an application if possible. When an input vector is applied, the output of the network is calculated and compared with the corresponding target vector. The error between the network output and the target is then fed back through the network to adjust the weights. The training is iterated until the error of the entire training set reaches an acceptably low level.

\subsection{Modelling Scalar Error Using the ANN}

The spindle thermal drifts mentioned in Section 3 were modelled again using the ANN technique. A 9-input (temperatures) and 3-output (thermal error components) multilayer perceptron ANN was used for the spindle thermal drifts in all three directions.

For network training, two important parameters should be determined: the number of hidden nodes and the learning rate (i.e. updating rate for the weights at every iteration). Too few hidden nodes probably will not allow the ANN to be trained well. However, too many hidden nodes will increase the difficulty of training and it usually tends to memorise everything including the noise. A small learning rate will increase the training time, but a large learning rate tends to make the training process oscillate or diverge.

In order to determine the necessary number of hidden nodes, several ANNs with different numbers of hidden nodes were trained. The results showed that for node number greater than 20 , the performance of the network did not improve further. Therefore, an ANN with a 9-20-3 structure ( 9 input nodes, 20 hidden nodes, and 3 output nodes) was selected for the empirical model of the spindle thermal drifts. The learning rate was started at a large value of 0.5 , but was continuously decreased during the iterative training so as to achieve a compromise between training speed and convergence. 
Because the output value of the sigmoid function is between 0 to 1 , all of the input and target vectors have been normalised between 0.1 to 0.9 . Normalising input patterns can be done in two ways, by normalising all of the input channels to a same factor or by normalising each input channel separately. The latter has the advantage of fully utilising the signals of every channel, but separate normalisation may also distort the relationships between inputs and cause more training time to be spent for recovering them. The former avoids the drawbacks of the later, but may not be trained as well as the latter in cases where some usable but small-magnitude channels are suppressed by a channel with a large magnitude.

It was found that normalising each channel separately gave better convergence accuracy than normalising all channels to the same factor for the spindle thermal drift prediction. The reason is probably that among the input temperatures, the spindle housing temperature is far larger than the machine column and leadscrew temperatures. Therefore, the temperature measurement of the spindle suppressed other temperature measurements when all channels were normalised to the same factor.

There are also two ways to update the weights: the pattern update algorithm (updating the weights per pattern passed) or the batch-update algorithm (updating the weights after all patterns in a training set are passed). Currently, there is no conclusion as to which algorithm is superior. It was found in this research that the pattern update algorithm gave a faster learning rate and smaller converged errors than the batchupdate algorithm for the spindle thermal drift prediction.

The training was done on a Macintosh IIx computer. The initial weights of the network were randomised between -0.3 and 0.3 . A relatively long training time was required to converge the errors to an acceptably low level. The averaged absolute errors after training were $6.5 \mu \mathrm{m}, 4.2 \mu \mathrm{m}$ and $6.0 \mu \mathrm{m}$ in the $x$-, $y$ - and $z$-directions, respectively. When compared with the errors using the MRA $(8.9 \mu \mathrm{m}, 3.8 \mu \mathrm{m}$ and $6.9 \mu \mathrm{m}$ in the $x$-, $y$ - and $z$ directions), the results were very close. Figures 10-12 compare the measured experimental data and the calculated output from the ANN model.

\subsection{Modelling Position-Dependent Error Using the ANN}

The 51 data sets of the $y$-axis thermal positioning errors were analysed again using the ANN technique. An ANN model with a 5-8-1 structure was used to establish the empirical model of the slope of the $y$-axis thermal positioning errors. Figure 13 compares the experimental results against the predictions using the model. It shows that the ANN can also fit the position-dependent thermal errors.

\section{Performance Comparisons}

Comparisons of the MRA and the ANN approaches were made from the following aspects:

1. The prediction accuracy for new observations.

2. Noise resistance robustness.

3. Fault tolerance.

\subsection{Prediction Accuracy for New Observations}

New observations of the spindle drift in the $z$-direction were collected from two tests. In the first test, the spindle was run continuously at a constant spindle speed of 1250 r.p.m. for six and half hours until it was thermally stabilised and then it was stopped to cool down. In the constant spindle speed test, the temperature field of the machine was continuously increased and then decreased. This test simulates cases for which several hours of continuous machining, inspection and set-up time are required, such as in the machining of large workpieces.

The second test was conducted at a random spindle speed. The machine was initially run at a constant spindle speed for $30 \mathrm{~min}$ and stopped for $15 \mathrm{~min}$. Then, the machine was set at another spindle speed and the same test cycle was repeated. The test was continued over 8 hours and the changes of the spindle speeds were selected randomly. The temperature field

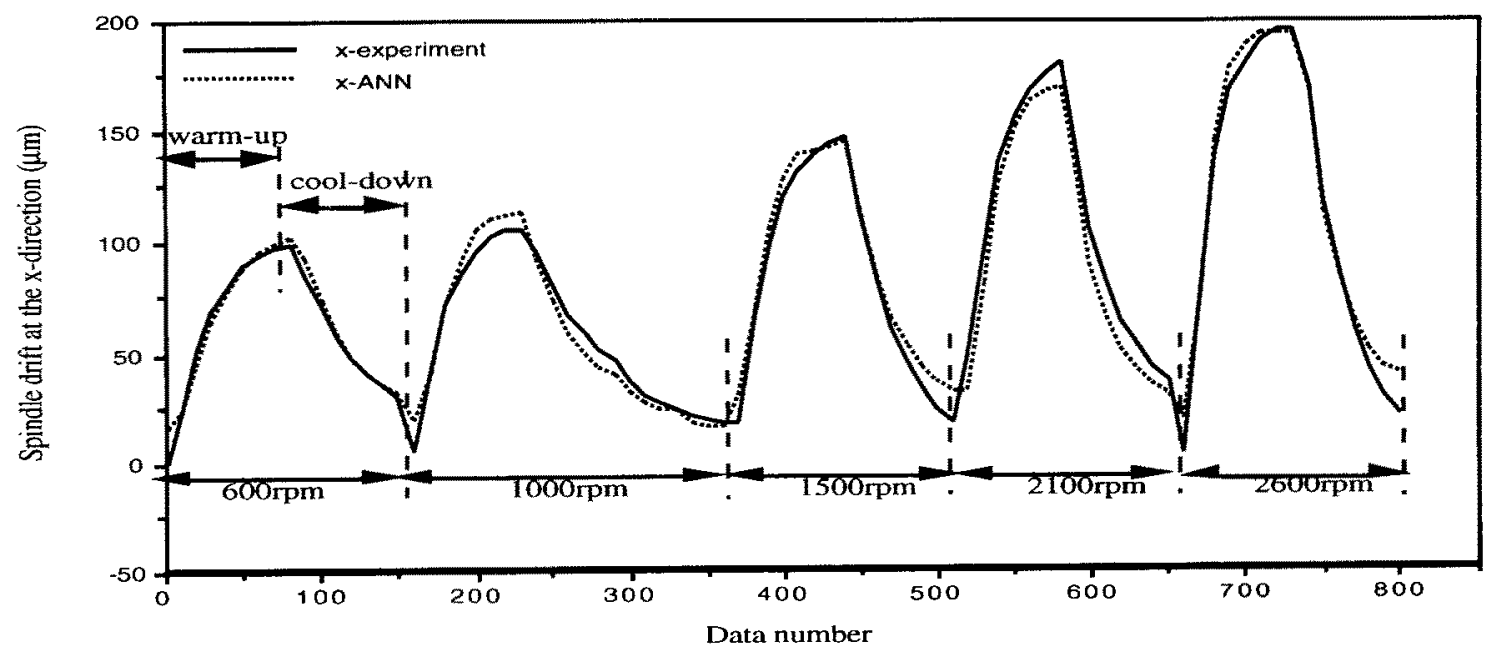

Fig. 10. The ANN modelling result of the spindle drift in the $x$-direction. 


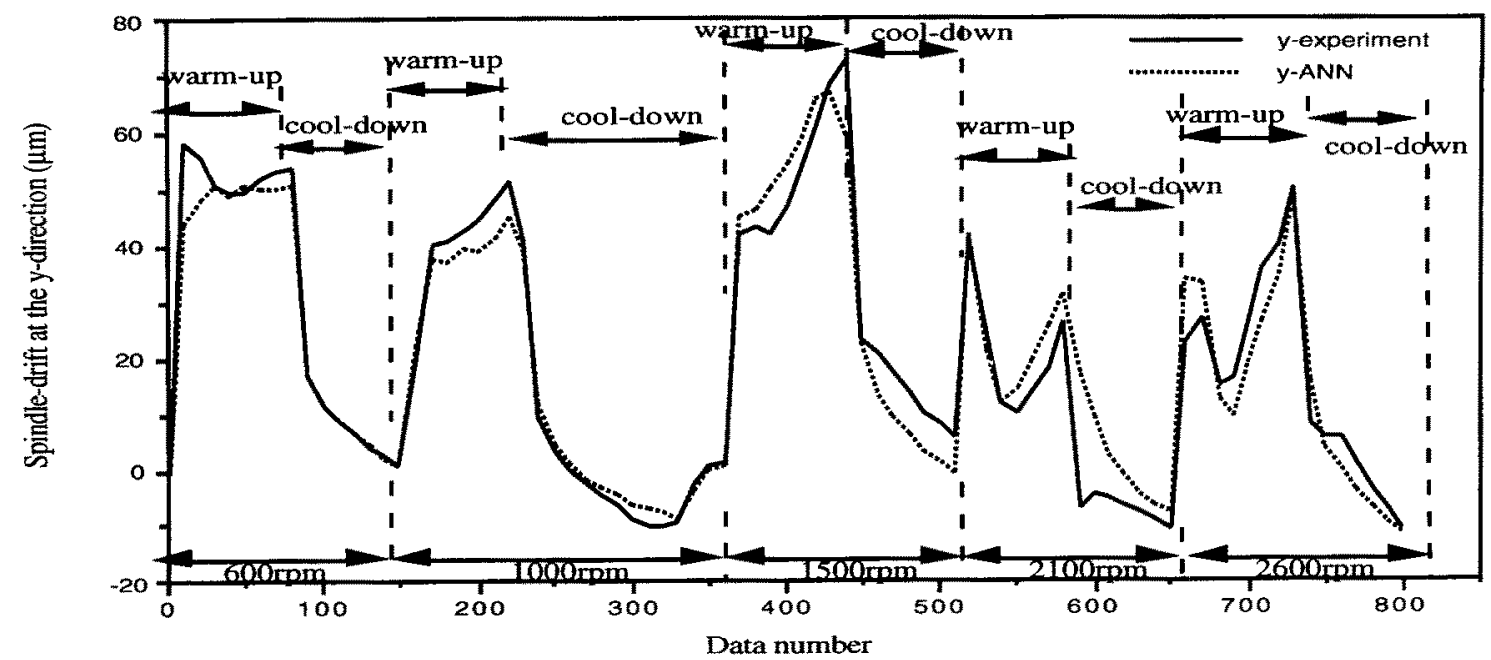

Fig. 11. The ANN modelling result of the spindle drift in the $y$-direction.

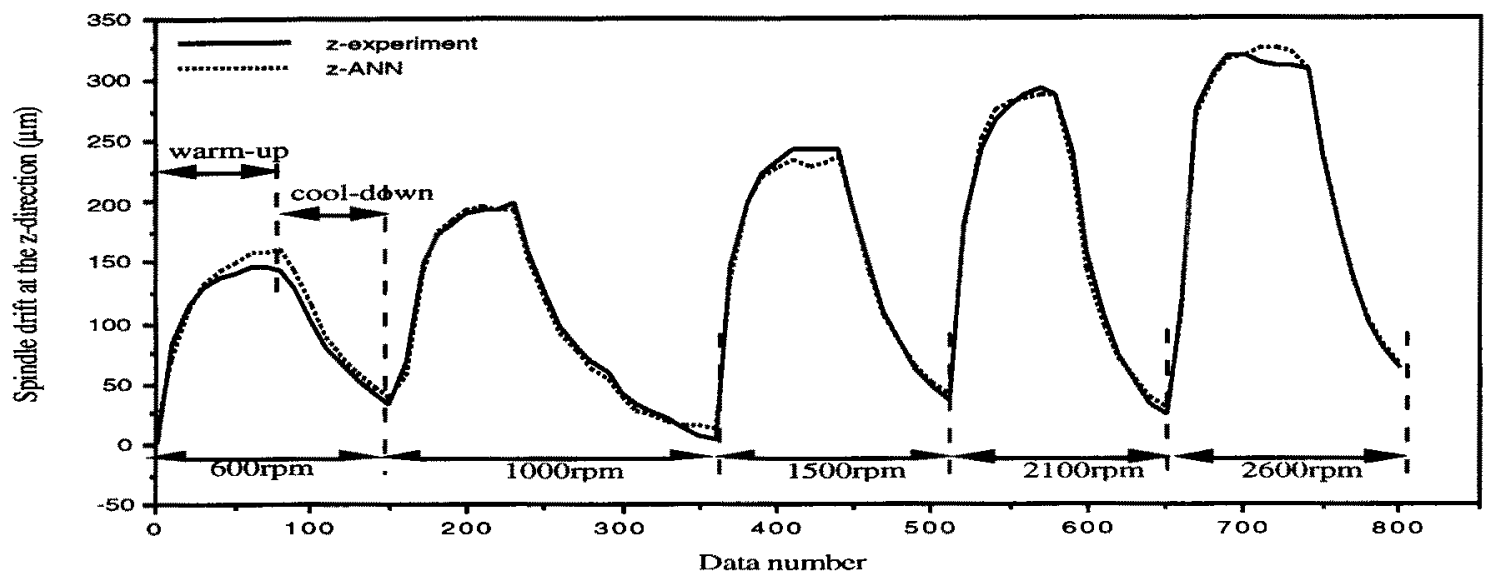

Fig. 12. The ANN modelling result of the spindle drift in the $z$-direction.
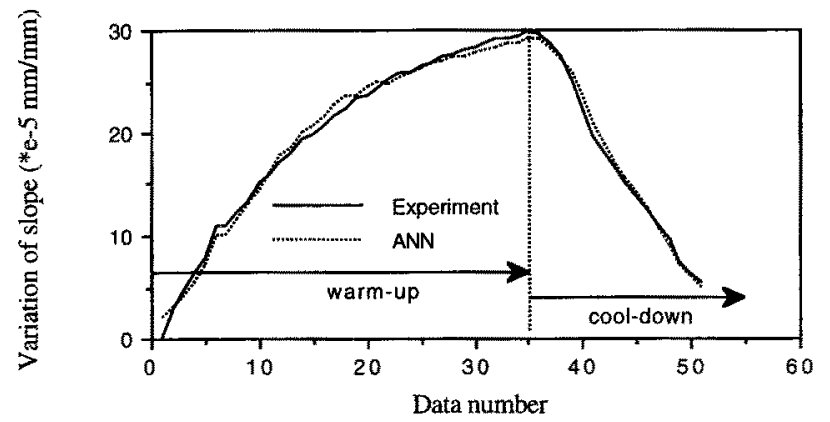

Fig. 13. The predicted and experimental results of the slope variation of the $y$-axis positioning errors.

of the machine fluctuated during the test. This test simulates cases with relatively short machining and set-up times.

Figures 14 and 15 compare the experimental data and predicted results using the MRA and the ANN approaches. They show that these two approaches are competitive and can predict the new observations almost equally well under varying spindle speeds and temperature history.

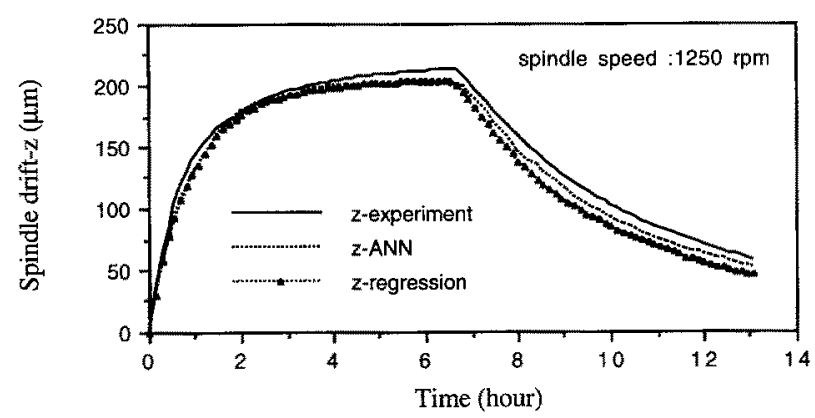

Fig. 14. The spindle thermal drift in the $z$-direction ( 1250 r.p.m.).

\subsection{Noise Resistance Robustness}

For the comparison of the noise resistance robustness, a $0.4^{\circ} \mathrm{C}$ noise level was added to all temperature measurements during the 1250 r.p.m. constant spindle speed test. This noise level was chosen because it included most noise levels of the temperature measurement system. Figure 16 shows that both approaches can predict well the spindle thermal growth under the $0.4^{\circ} \mathrm{C}$ noise level. 


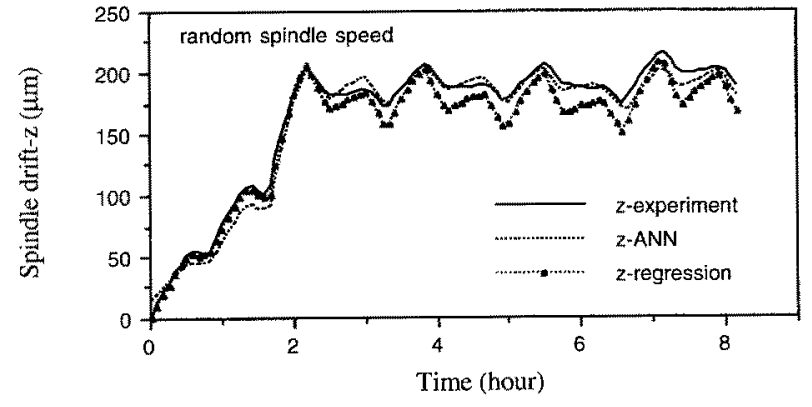

Fig. 15. The spindle drift in the $z$-direction (random spindle speed).

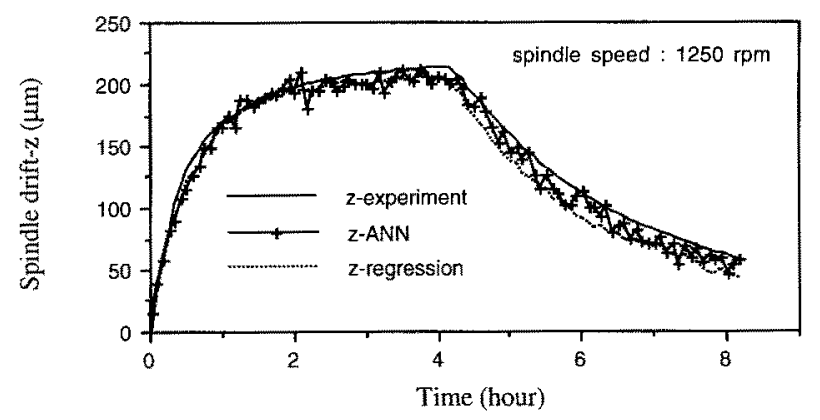

Fig. 16. The noise effect in the $z$-spindle thermal drift ( 1250 r.p.m.).

\subsection{Fault Tolerance}

To compare the fault tolerance of the MRA and the ANN approaches, the temperature rise at the spindle, one of the most important temperature measurements, was cut off. Figure 17 compares the experimental data and the modelling results of the spindle drift in the $x$-direction using the MRA and the ANN approaches. It clearly shows that the ANN has better fault tolerance capability than the MRA approach. The increased fault tolerance of the ANN is probably due to the fact that the output of every neuron in an ANN is the combination of various inputs. Therefore, a hardware failure in one of the thermocouples is not usually catastrophic, because the other inputs can still stimulate a percentage of output.

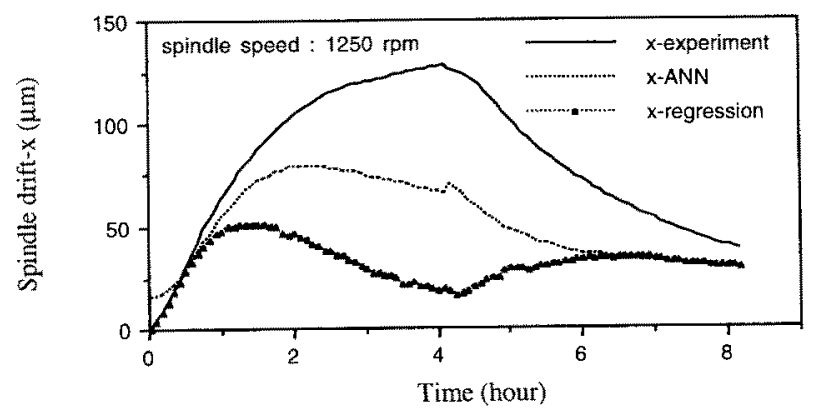

Fig. 17. Comparisons of the fault tolerance capabilities of the MRA and the ANN approaches.

\section{Conclusions}

1. Some thermal volumetric error components are not only time-variant but also position-dependent. A strategy for formulating both the scalar and position-dependent error components is proposed. The major advantage of this new modelling strategy is that it greatly reduces the number of empirical models needed to predict the position-dependenterrors. It can also accommodate the non-rigid body kinematic effects and nonlinear properties.

2. The multiple regression analysis and multiple-layer perceptron neural network approaches have been applied for the real-time prediction of thermal errors. Both approaches adopt a multi-input method to accommodate the different thermal time constants of the various machine elements. The test results have shown that both models can predict well the new observations of thermal errors under varying spindle speeds and varying temperature history.

3. Both approaches use a computerised algorithm to automatically search for the nonlinear and interaction terms of the multiple temperature measurements in an empirical model. In the MRA approach, the stepwise regression analysis is proposed to select the higher-order and cross-product terms of temperature measurements based on statistical criteria. For the ANN approach, the nonlinear and interaction characteristic is recognised by a supervised backpropagation learning procedure.

4. Comparisons of the MRA and ANN approaches showed that their prediction accuracy for new observations was competitive. They both produced good predictions for new observations when a $0.4^{\circ} \mathrm{C}$ noise level was added to temperature measurements. In terms of the fault tolerance capability, the ANN was better than the MRA when one of the temperature measurements was cut off.

5. The proposed approaches have been implemented in a 3axis horizontal machining centre [6]. Evaluated by an HP laser interferometer measurement system, the machine accuracy has been improved by a factor of ten. Also, the CMM inspection of a cut aluminium workpiece has shown that dimensional errors owing to the thermal expansions of the $y$-axis are reduced from $92.4 \mu \mathrm{m}$ to $7.2 \mu \mathrm{m}$ and the depth difference of milled surfaces owing to the spindle thermal growth is reduced from $196 \mu \mathrm{m}$ to $8 \mu \mathrm{m}$.

\section{References}

1. M. H. Attia and L. Kops, "Nonlinear thermoelastic behavior of structural joints-solution to a missing link for predication of thermal deformation of machine tools", Journal of Engineering for Industry, 101, pp. 348-354, August 1979.

2. J. Janeczko, "Machine tool thermal distortion compensation", 4th Biennial International Machine Tool Technology Conference, September 1988.

3. J. X. Sha, "Spindle thermal error measurement and modeling on a CNC lathe". Technical Report, MEAM Dept, University of Michigan, April 1990.

4. M. A. Woytowitz et al. "Tool path error analysis for high precision milling with a magnetic bearing spindle", ASME pp. 129-142, 1989. 
5. A. Kurtoglu, "The accuracy improvement of machine tools", Annals of the CIRP, 39(1), pp. 417-419, 1990.

6. J. S. Chen, J. Yuan, J. Ni and S. M. Wu, "Real-time compensation of time-variant volumetric errors on a machining center", Journal of Engineering for Industry, 115, pp. 472-479, 1993.

7. M. A. Donmez, D. S. Blomquist, R. J. Hocken, C. R. Liu and M. M. Barash, "A general methodology for machine tool accuracy enhancement by error compensation", Precision Engineering, 8
(4), pp. 187-196, October 1986.

8. J. S. Chen, J. Yuan, J. Ni and S. M. Wu, "Compensation of nonrigid body kinematic effect on a machining center", Transactions of the North American Manufacturing Research Institute of SME, 20, pp. 325-330, 1992.

9. D. E. Rumelhart, G. E. Hiton and R. J. Williams, "Learning internal representations by error propagation", Parallel Distributed Processing, 1, pp. 318-362, MIT Press, Cambridge, MA, 1986. 